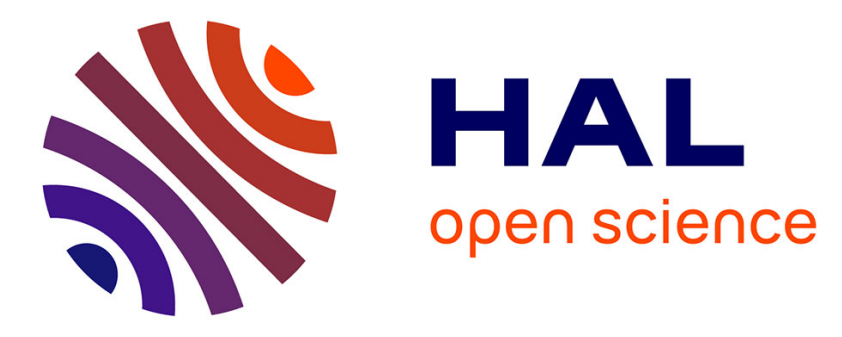

\title{
Investigation of the Self-Doping Process in HgSe Nanocrystals
}

Clément Livache, Bertille Martinez, Adrien Robin, Nicolas Goubet, Benoit

Dubertret, Hongyue Wang, Sandrine Ithurria, Hervé Aubin, Emmanuel Lhuillier

\section{To cite this version:}

Clément Livache, Bertille Martinez, Adrien Robin, Nicolas Goubet, Benoit Dubertret, et al.. Investigation of the Self-Doping Process in HgSe Nanocrystals. E-MRS, May 2017, strasbourg, France. pp.1700294, 10.1002/pssa.201700294 . hal-01587739

\section{HAL Id: hal-01587739 \\ https://hal.science/hal-01587739}

Submitted on 10 Jul 2020

HAL is a multi-disciplinary open access archive for the deposit and dissemination of scientific research documents, whether they are published or not. The documents may come from teaching and research institutions in France or abroad, or from public or private research centers.
L'archive ouverte pluridisciplinaire HAL, est destinée au dépôt et à la diffusion de documents scientifiques de niveau recherche, publiés ou non, émanant des établissements d'enseignement et de recherche français ou étrangers, des laboratoires publics ou privés. 


\title{
Investigation of the self-doping process in HgSe nanocrystals
}

\author{
Clément Livache ${ }^{1,2}$ Bertille Martinez, ${ }^{1,2}$ Adrien Robin, ${ }^{2}$ Nicolas Goubet ${ }^{1,2}$ Benoit Dubertret $^{2}$, Hongyue \\ Wang ${ }^{2}$, Sandrine Ithurria ${ }^{2}$, Hervé Aubin ${ }^{2}$, Emmanuel Lhuillier $^{{ }^{*}}$ \\ ${ }^{1}$ Sorbonne Universités, UPMC Univ Paris 06, CNRS-UMR 7588, Institut des NanoSciences de Paris, 4 place \\ Jussieu, 75005 Paris, France \\ ${ }^{2}$ Laboratoire de Physique et d'Etude des Matériaux, ESPCI-ParisTech, PSL Research University, \\ Sorbonne Université UPMC Univ Paris 06, CNRS, 10 rue Vauquelin 75005 Paris, France.
}

\begin{abstract}
Colloidal nanocrystals are an interesting platform for the design of low cost infrared optoelectronic materials. In addition to conventionally observed interband features, doped nanocrystals present intraband transitions, which expand the possibilities for electronic spectrum engineering. In this paper, we review recent results obtained in the field of mercury chalcogenides self-doped nanocrystals presenting absorption features in the mid infrared range. In particular, we discuss the results relative to the synthesis, control of doping and transport properties of HgSe colloidal nanocrystals.
\end{abstract}

Keywords: HgSe, self-doping, colloidal quantum dot, electrolyte gating, photoresponse, infrared.

*To whom correspondence should be addressed. E-mail: el@insp.upmc.fr 


\section{Introduction}

Colloidal quantum dots (CQD) are offering an alternative platform to revisit optoelectronics [1] while combining high performances and low fabrication cost. In addition to display and solar cell [2], the use of CQD for infrared optoelectronics is attracting more and more interest [3-5]. To achieve narrow energy transition as those required to get absorption in the mid infrared, narrow band gap materials [6] can be used. Among them mercury chalcogenides [7] $(\mathrm{HgX})$ have certainly reached the highest maturity in this field. Indeed, $\mathrm{HgX}$ [8] benefits from the II-VI semiconductor maturity in term of colloidal synthesis as well as the vicinity from the well-established bulk $\mathrm{HgCdTe}$ alloy. To this day, $\mathrm{HgX}$ are the only materials to combine tunable optical features from telecom wavelengths [9] up to the $\mathrm{THz}$ [10] range with photoconductive properties. Over the last three years several groups have reported the integration of $\mathrm{HgX}$ nanocrystals as active material for infrared detection with competitive level of performances [4]. Recent development include new surface chemistries, the switch from photoconductor to photovoltaic [11] or phototransistor [12], multispectral device [13,14], the integration of plasmonic functionalities [15-17], hybridization with graphene [18,19] and even the first demonstration of mid-IR CQD based focal plane array [20].

While HgTe behaves as an intrinsic material for which the mid-IR transition is an interband transition, HgS [21-23] and HgSe $[10,24,25]$ present self-doping properties. As a result, they come under a stable $n$-doped form with intraband transition within the conduction band. Intraband transition in doped quantum wells has been used since the late 80 's as an alternative path for the design of complex heterostructures, including quantum well infrared photodetectors [26] and sources such as quantum cascade lasers [27]. In such materials, metallurgic design which was so far the most common way to achieve band gap engineering is replaced by wave-function engineering where geometric parameters determine the heterostructure spectrum. Moreover, as these materials are unipolar materials, no management of surface recombination center is necessary. In this sense, the introduction of intraband/intersubband transition is offering a new degree of freedom for the design of infrared devices, and so doping becomes a key driving parameter which needs to be accurately controlled. Up to now, the II-VI colloidal HgX compounds are not yet achieving the maturity of III-V heterostructures for the design of optoelectronic devices. In this short review, we discuss some of the recent results obtained by our group on this question. The origin of doping and path to control its magnitude are especially discussed. Finally we show how the doping impacts the transport properties of thin films of HgSe CQD, down to the single particle level [28].

\section{Discussion}

$\mathrm{HgSe}$, as HgTe, presents an inverted band structure [29], where the band with a $\Gamma_{6}$ geometry presents a lower energy than the band with a $\Gamma_{8}$ geometry, see Figure $1 \mathrm{a}$. Interband transition occurs between the two $\Gamma_{8}$ bands. Under bulk form, $\mathrm{HgSe}$ is a semimetal: this aspect is the most interesting for the design of optical features at long wavelength, since by tuning the confinement energy, any wavelength can potentially be adressed.

We demonstrate a growth method for HgSe based on the reaction of mercury oleate in oleylamine with a selenium precursor at moderate temperature $\left(60-120^{\circ} \mathrm{C}\right.$ ). By choosing the right Se precursor (Se complexed with trioctylphosphine or $\mathrm{SeS}_{2}$ disolved in oleylamine), a large size tunability can be achieved 
from $3 \mathrm{~nm}$ up to $50 \mathrm{~nm}$, see Figure 2a-b. The absorption spectra of the HgSe CQD present two characteristic features in the infrared range. At high energy, a broad feature is the result of interband transition. At low energy $(\lambda>3 \mu \mathrm{m})$ a narrow absorption peak is the result of intraband transition, see Figure $1 \mathrm{~b}$-c. Thanks to the large size tunability, the wavelength associated to the mid IR feature can be adjusted from 3 to $26 \mu \mathrm{m}$ [10,30]. Similar mid-IR transition where previously observed in optically [31] and electrochemically charged [32] wide band gap CQD such as CdSe. Here the key difference is the fact that the stable form of the HgSe CQD is the negatively charged form. This is a key breakthrough for the design of infrared detector, since it drastically simplify the device design.
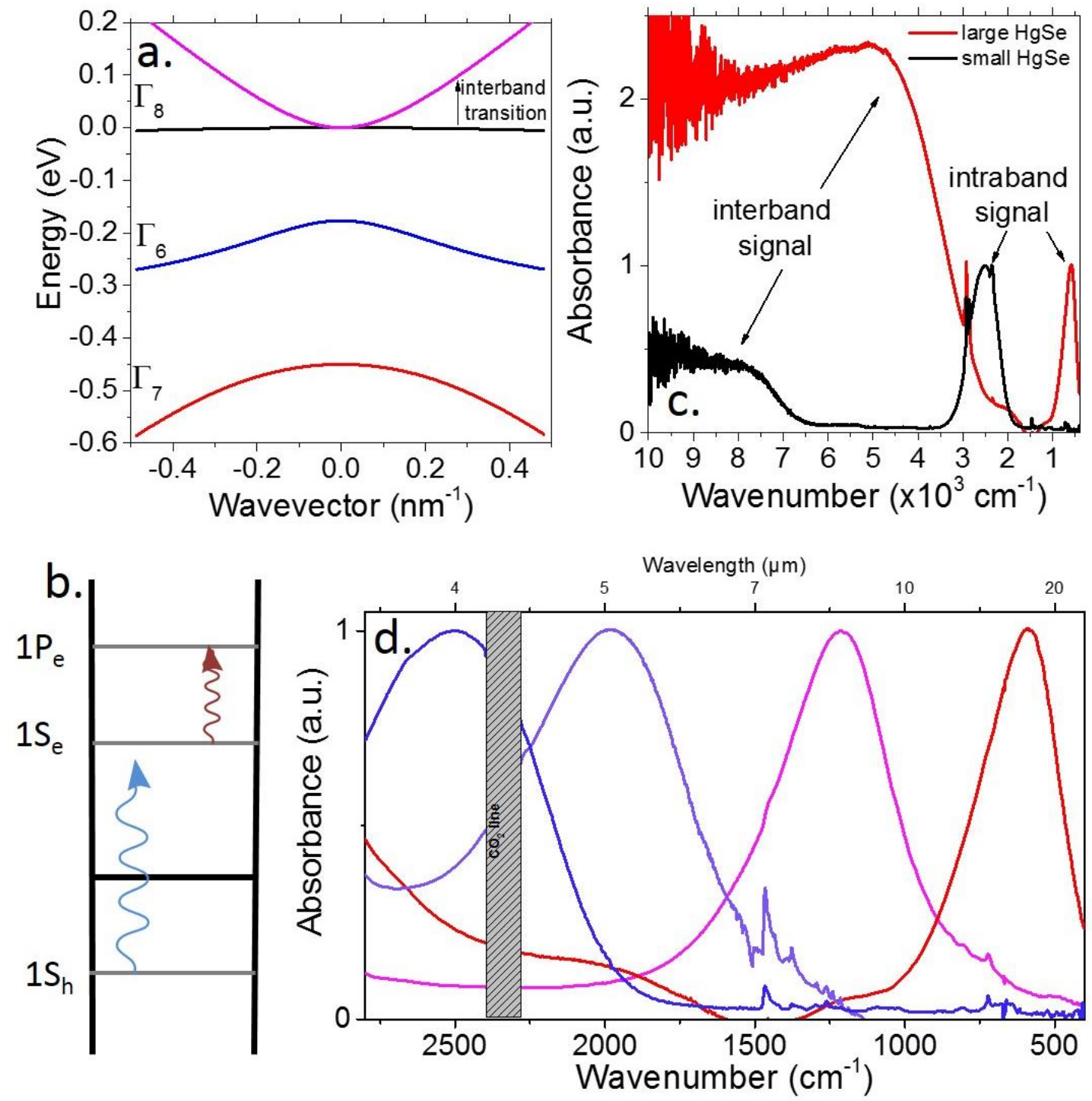

Figure 1 a. Band structure of HgSe. b. Schematic view of confined HgSe CQD with interband and intraband transistions. c. Absorption spectra for two sizes of HgSe CQD. $d$. Zoom on the part relative to the intraband component of the absorption spectra of four sizes of HgSe CQD. Adapted from reference [10]. 
The main hope raised by these material is the design of low cost devices, in particular low cost infrared sensors such as focal plane arrays (FPA) in the 100 to 1000 range. Such FPA typically answer new needs in the field of IR detection such as assistance for night driving or for the thermal management of buildings. If mass scale application is targeted, the synthesis of large batch of these materials is critical. We demonstrated the growth of HgSe CQD up to $10 \mathrm{~g}$ of dried material. This number has to be compared with the amount of material that is used for a single device, typically around $10 \mu \mathrm{g}$, which means that the synthesis of colloidal materials we developed can be used for the production of a million devices. One may also argue that the toxicity of this material might be a deal breaker for the use of CQD in mass scale market. However, the field of IR detection strongly differs from solar cell. Indeed in the solar cell field, the goal is to produce $>1 \mu \mathrm{m}$ thick film over $\mathrm{km}^{2}$ which requires tons of material. Here the amount of material to introduce a CQD based IR detector in each car sold in Europe per year ( $\approx 20$ millions units per year) is about $200 \mathrm{~g}$. So even if we include a $90 \%$ waste factor at the fabrication scale, the amount of active material remains small $(\approx 2 \mathrm{~kg})$, far below the amount of lead in a single lead based battery.

Another key aspect regarding this material is its stability, andin particular its ability to sustain a clean room processing, where the material is exposed to high temperature baking step or to exposition to nonsolvent. As a first step to address this issue, we demonstrate the processability of an HgSe film under pixel form, see Figure 2c. The obtained pixel presents sharp edges for size down to $20 \mu \mathrm{m}$.

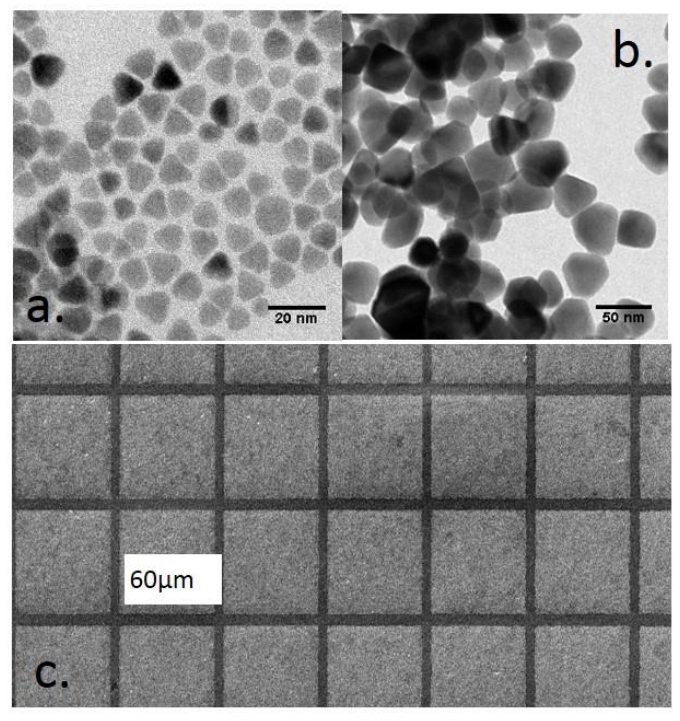

Figure $2 a$. and b. are TEM images of two sizes of HgSe CQD. c. SEM image of a film of HgSe CQD after its processing under pixel form. Adapted from reference [10].

The growth of HgX CQD has been strongly improved over the recent years [4,33]. However, to integrate this material in an infrared sensor, the doping in layers of $\mathrm{HgS}(\mathrm{e})$ must be well controlled. Understanding the doping origin and demonstrating paths to control its magnitude are of utmost importance. One way of controlling the doping level is shown on Figure 3a: as ligand exchange is conducted on a film of HgSe CQD, the absorption spectrum is strongly affected: at the end of the synthesis, the nanocrystals are 
capped with dodecanethiol (DDT) ligands, and the intraband signal prevails over the interband contribution. When these DDT ligands are replaced by shorter ligands, we observe little by little the appearance of a peak relative to the $1 S_{h}-1 S_{e}$ interband transition and the relative magnitude of the intraband peak decreases [34]. This results from the displacement of the Fermi level from above the 1s state to an almost $1 \mathrm{~s}$ resonant level. By fitting the relative weight of the interband and intraband contributions, we can estimate the population per CQD: it typically decreases from 2 electrons with DDT to around 0.2 electron per CQD in the case of $\mathrm{S}^{2-}$ capping, see Figure $3 \mathrm{~b}$. The mechanism at the origin of the relative displacement of the Fermi level with respect to the $1 \mathrm{~s}$ state is explained on the schematic Figure 3c. The dipole associated with the ligand induces a band bending at the surface of the CQD [35,36] and shifts the conduction band towards the vacuum level. While the core of the CQD is doped, the external part is depleted due to the electric field resulting from the mercury to ligand bond. The origin of the doping itself is explained on the Figure 3d. $\mathrm{HgX}$ CQD combine a large work function [37] with a narrow band gap nature, which makes possible that the conduction band energy is below the environment redox potential. In other words, where most CQD are oxidized by the environment, HgSe and HgS CQD are reduced by the environment and their stable form is negatively charged. This explains why the intraband absorption is a robust property and stays unaffected after several month spent in air environment.

For larger CQD, the narrower band gap nature makes that the reduction by the environment is even more important [23]. It has been show in the case of $\mathrm{HgS}$ that higher order states get filled [38] and that little by little the transition switches from its intraband behavior to a plasmonic behavior [21].
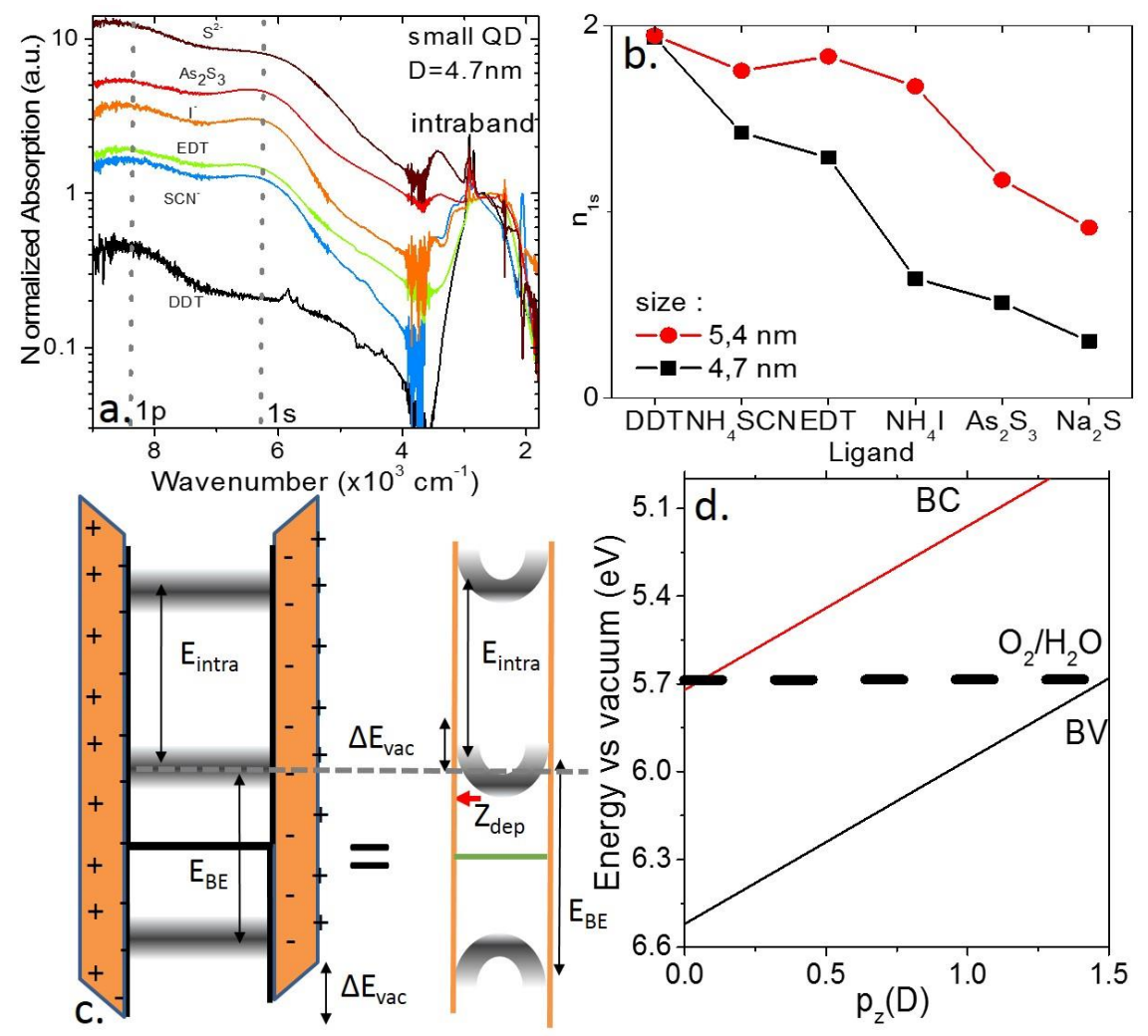

Figure 3 a. Absorption spectra normalized at the intraband contribution for small HgSe CQD capped with different ligands. b. Population for two sizes HgSe CQD capped with different ligands. $c$. Schematic of the 
band bending occuring at the nanocrystal surface resulting from the presence of ligands. $d$. Spectrum of $\mathrm{HgSe} C Q D$ as a function of the surface dipole magnitude. Adapted from reference [30].

Within mind the integration of this material for photodetection, the investigation of transport properties is as important as the careful design of the optical spectrum. To do so, we investigate the conduction in an ion-gel electrolytically gated transistor configuration $[39,40]$. The latter presents several advantages: (i) the transistors are air operable; (ii) the gate capacitance is large and allows to inject up to several electrons per CQD, which is critical to tune the carrier density of a nanocrystal which already includes in its native state 2 electrons. Finally (iii) electrolytic gating can be used to gate thick films which is convenient for the design of phototransistors [14]. In practice, we first conduct a ligand exchange toward $A s_{2} S_{3}$ capping: this surface chemistry is transparent in the infrared range and leads to a strong inter QCD coupling [41,42]. In a second step, ion gel electrolyte made of $\mathrm{LiClO}_{4}$ dissolved in polyethylene glycol is brushed on the top of the film. For a scheme of the device, see Figure $4 \mathrm{a}$.
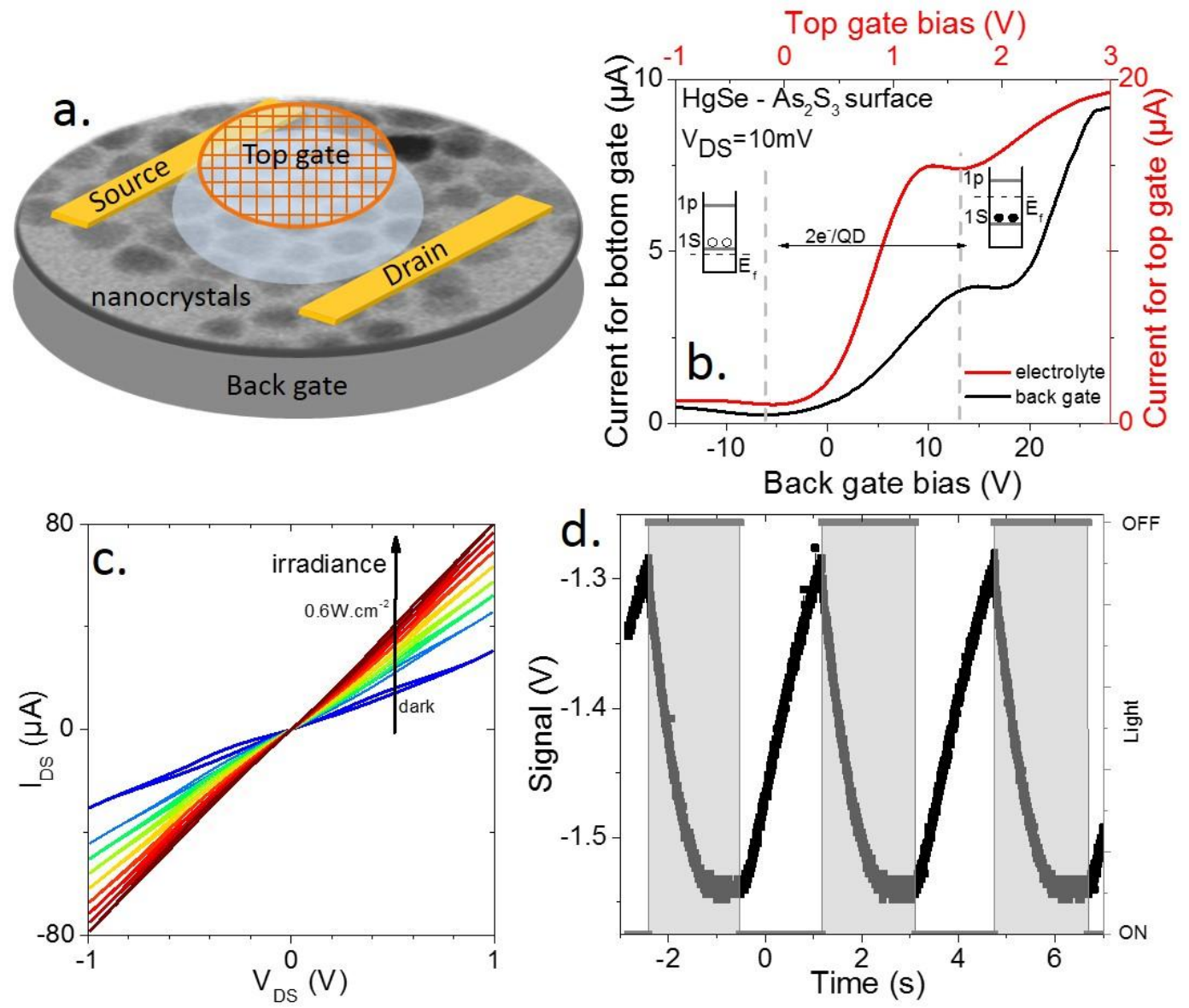

Figure 4a. Schematic of the ion gel electrolyte gated transistor, which channel is made of HgSe CQD. $b$ Transfer curve (ie drain current as a function of gate voltage) for a thin film of HgSe CQD. Adapted from reference [10]. C IV curve for thin film of HgSe CQD capped with $S^{2-}$ ligands under dark condition and under illumination by a $1.55 \mu \mathrm{m}$ laser diode at different power. D. current modulation for thin film of HgSe CQD capped with $S^{2-}$ ligands as a function of time while light is turned on and off. 
The transfer curve obtained from the described transistor presents a conductance increasing as electron are injected (positive gate bias), which is characteristic of an $n$-type material, see Figure $4 \mathrm{~b}$. This further confirms the electron doping of HgSe CQD. The rise in conductance is moreover non monotonic and presents a local minimum around $1.5 \mathrm{~V}$ for the top electrolytic gate. This behavior is the result of the Pauli blockade: as electrons are injected, some CQD have their 1s state fully filled (2 electrons) and transport has to occur through the $1 p$ state which requires to pass an activation energy equal to the intraband transition. This results in a drop of the differential conductance.

If $\mathrm{S}^{2-}$ ligands are used the $1 \mathrm{~s}$ state of the conduction band is almost empty and the material can be used as an interband photodetector [34]. Under illumination the film present a positive photoresponse which has been estimated to be in the $10-20 \mathrm{mAW}^{-1}$ for an illumination at $1.55 \mu \mathrm{m}$ and room temperature operation, see Figure 4c.A key limitation of HgSe compared to HgTe [43] is currently its slow photoreponse, with a typical time response of several seconds, see Figure $4 \mathrm{~d}$.

Another key interesting question regarding these mid IR nanoparticles relates to the investigation of their properties at the single particle level, in particular to investigate the origin of the broadening of the intraband transition. This question is far more challenging than for wide band gap nanoparticles. Indeed, most of the experimental setup based on optical measurements cannot be used in the mid IR because IR CQD presents a low PL efficiency and because experimental setups get more complex/less efficient. As a result, new experimental paths have to be developed. We recently proposed an approach based on an on-chip tunnel spectroscopy $[28,44]$. The basic idea is to electrospray a solution of CQD onto a chip of nanogap. The latter are prepared in clean room and present a spacing compatible with the CQD size. At each step of the evaporation, the conductance of the nanogap is tested. The spray is interrupted when a sudden increase of the nanogap channel conductance is observed, see Figure 5a-d for a schematic of the setup. The sample is then transferred in the cryostat and characterized at low temperature. 

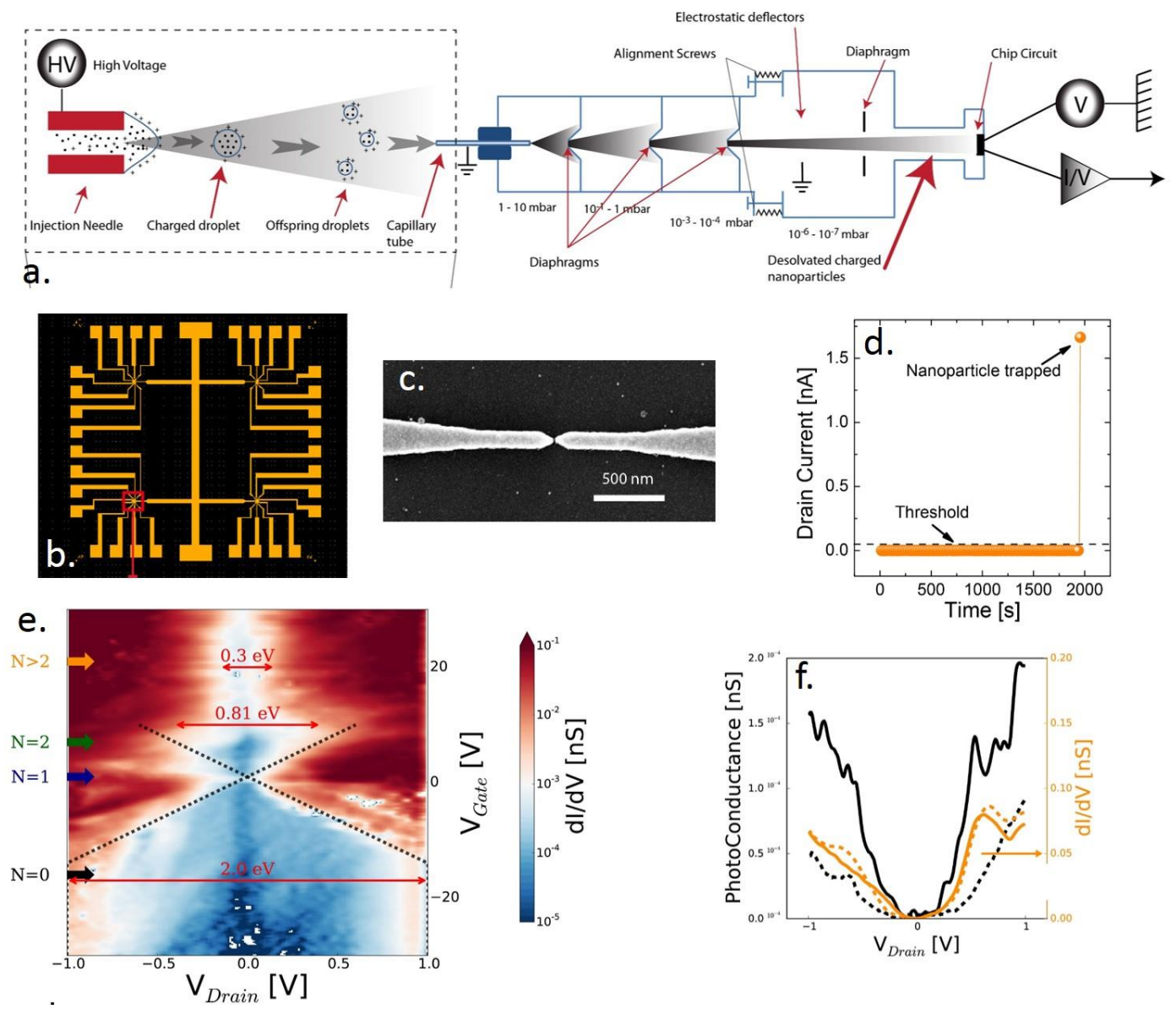

Figure 5a. Schematics of the spray method for the deposition of a single HgSe CQD onto a tunnel nanogap. b. Image of the chip with 32 nanogaps. c. SEM image of the nanogap. $d$. Current as a function of the time as the HgSe CQD solution is sprayed on the nanogap chip. e. Tunnel map for a single HgSe. The map gives the tunnel conductance as a funtion of drain and gate bias. f. Photoconductance (black lines) and conductance (orange lines) measured under illumination (continuous lines) and in the dark (dash lines), as the applied bias is adapted from reference [16].

A typical tunnel map is given in Figure 5e. Two tunnel gaps are observed in this tunnel spectrum: one wide band gap around $2 \mathrm{eV}$ and one narrower band gap of $0.85 \mathrm{eV}$ at a higher gate filling. Once we take into account the fact that the bias is equally applied over the two sides of the nanogap and that Coulomb polarization energy is of the order of $80 \mathrm{meV}$ [16], we obtain two excitation gaps of $0.85 \mathrm{eV}$ and $0.25 \mathrm{eV}$. The latter corresponds to the interband and intraband transition determined using ensemble absorption measurements. This measurement confirms that the doping is already presents at the single particle level. Finally, we have also been able to measure photoconduction of a single nanocrystal, see Figure 5 and demonstrate a large photogain up to 50.

\section{Conclusion}


We investigated the synthesis of HgSe nanocrystals. By tuning their size between 3 and $50 \mathrm{~nm}$, we have been able to tune their absorption in the mid IR from 3 to $26 \mu \mathrm{m}$ peak. The doping of the material is the result of reduction by the environment but can be further finely control using the CQD surface chemistry. Finally, transport measurements confirm the $n$-doping of the CQD, including at the single particle scale.

\section{Acknowledgments}

We thank Agence Nationale de la Recherche for funding through grant Nanodose and H2DH. This work has been supported by the Region Ile-de-France in the framework of DIM Nano-K through the grant dopQD. This work was supported by French state funds managed by the ANR within the Investissements d'Avenir programme under reference ANR-11-IDEX-0004-02, and more specifically within the framework of the Cluster of Excellence MATISSE.

\section{References}

[1] D. V. Talapin, J.-S. Lee, M. V. Kovalenko, and E. V. Shevchenko, Chem. Rev. 110, 389 (2010).

[2] G. H. Carey, A. L. Abdelhady, Z. Ning, S. M. Thon, O. M. Bakr, and E. H. Sargent, Chem. Rev. 115, 12732 (2015).

[3] S. Keuleyan, E. Lhuillier, V. Brajuskovic, and P. Guyot-Sionnest, Nat. Photonics 5, 489 (2011).

[4] E. Lhuillier and P. Guyot-Sionnest, IEEE J. Sel. Top. Quantum Electron. 23, 6000208 (2017).

[5] E. Lhuillier, S. Keuleyan, H. Liu, and P. Guyot-Sionnest, Chem. Mater. 25, 1272 (2013).

[6] S. V. Kershaw, A. S. Susha, and A. L. Rogach, Chem. Soc. Rev. 42, 3033 (2013).

[7] E. Lhuillier, S. Keuleyan, P. Rekemeyer, and P. Guyot-Sionnest, J. Appl. Phys. 110, 33110 (2011).

[8] S. Keuleyan, E. Lhuillier, and P. Guyot-Sionnest, J. Am. Chem. Soc. 133, 16422 (2011).

[9] H. Seong, K. Cho, and S. Kim, Semicond. Sci. Technol. 23, 75011 (2008).

[10] E. Lhuillier, M. Scarafagio, P. Hease, B. Nadal, H. Aubin, X. Z. Xu, N. Lequeux, G. Patriarche, S. Ithurria, and B. Dubertret, Nano Lett. 16, 1282 (2016).

[11] P. Guyot-Sionnest and J. A. Roberts, Appl. Phys. Lett. 107, 253104 (2015).

[12] M. Chen, H. Lu, N. M. Abdelazim, Y. Zhu, Z. Wang, W. Ren, S. V. Kershaw, A. L. Rogach, and N. Zhao, ACS Nano (2017).

[13] X. Tang, X. Tang, and K. W. C. Lai, ACS Photonics 3, 2396 (2016).

[14] E. Lhuillier, A. Robin, S. Ithurria, H. Aubin, and B. Dubertret, Nano Lett. 14, 2715 (2014).

[15] Y. Yifat, M. Ackerman, and P. Guyot-Sionnest, Appl. Phys. Lett. 110, 41106 (2017).

[16] X. Tang, G. fu Wu, and K. W. C. Lai, J. Mater. Chem. C (2016).

[17] M. Chen, L. Shao, S. V. Kershaw, H. Yu, J. Wang, A. L. Rogach, and N. Zhao, ACS Nano 8, 8208 (2014).

[18] X. Tang, G. F. Wu, and K. W. C. Lai, Appl. Phys. Lett. 110, 241104 (2017).

[19] N. Huo, S. Gupta, and G. Konstantatos, Adv. Mater. 29, 1606576 (2017). 
[20] C. Buurma, R. E. Pimpinella, A. J. Ciani, J. S. Feldman, C. H. Grein, and P. GuyotSionnest, Proc. SPIE 9933, 993303 (2016).

[21] G. Shen and P. Guyot-Sionnest, J. Phys. Chem. C 120, 11744 (2016).

[22] K. S. Jeong, Z. Deng, S. Keuleyan, H. Liu, and P. Guyot-Sionnest, J. Phys. Chem. Lett. 5, 1139 (2014).

[23] B. Yoon, J. Jeong, and K. S. Jeong, J. Phys. Chem. C 120, 22062 (2016).

[24] Z. Deng, K. S. Jeong, and P. Guyot-Sionnest, ACS Nano 8, 11707 (2014).

[25] J. Jeong, B. Yoon, Y.-W. Kwon, D. Choi, and K. S. Jeong, Nano Lett. 17, 1187 (2017).

[26] H. Schneider and H. C. Liu, Quantum Well Infrared Photodetectors: Physics and Applications (Springer, 2006).

[27] J. Faist, F. Capasso, D. L. Sivco, C. Sirtori, A. L. Hutchinson, and A. Y. Cho, Science 264, 553 (1994).

[28] H. Wang, E. Lhuillier, Q. Yu, A. Zimmers, B. Dubertret, C. Ulysse, and H. Aubin, ACS Nano 11, 1222 (2017).

[29] E. Lhuillier, S. Keuleyan, and P. Guyot-Sionnest, Nanotechnology 23, 175705 (2012).

[30] B. Martinez, C. Livache, L. D. N. Mouafo, N. Goubet, H. Crugel, S. Ithurria, H. Aubin, A. Ouerghi, B. Doudin, B. Dubertret, M. G. Silly, R. P. S. M. Lobo, J.-F. Dayen, and E. Lhuillier, Submitted (2017).

[31] S. Keuleyan, J. Kohler, and P. Guyot-Sionnest, J. Phys. Chem. C 118, 2749 (2014).

[32] M. Shim and P. Guyot-Sionnest, Nature 407, 981 (2000).

[33] E. Izquierdo, A. Robin, S. Keuleyan, N. Lequeux, E. Lhuillier, and S. Ithurria, J. Am. Chem. Soc. 138, 10496 (2016).

[34] A. Robin, C. Livache, S. Ithurria, E. Lacaze, B. Dubertret, and E. Lhuillier, ACS Appl. Mater. Interfaces 8, 27122 (2016).

[35] M. Soreni-Harari, N. Yaacobi-Gross, D. Steiner, A. Aharoni, U. Banin, O. Millo, and N. Tessler, Nano Lett. 8, 678 (2008).

[36] P. R. Brown, D. Kim, R. R. Lunt, N. Zhao, M. G. Bawendi, J. C. Grossman, and V. Bulović, ACS Nano 8, 5863 (2014).

[37] A. H. Nethercot, Phys. Rev. Lett. 33, 1088 (1974).

[38] M. Chen and P. Guyot-Sionnest, ACS Nano (2017).

[39] E. Lhuillier, S. Ithurria, A. Descamps-Mandine, T. Douillard, R. Castaing, X. Z. Xu, P.-L. Taberna, P. Simon, H. Aubin, and B. Dubertret, J. Phys. Chem. C 119, 21795 (2015).

[40] E. Lhuillier, J.-F. Dayen, D. O. Thomas, A. Robin, B. Doudin, and B. Dubertret, Nano Lett. 15, 1736 (2015).

[41] E. Lhuillier, S. Keuleyan, P. Zolotavin, and P. Guyot-Sionnest, Adv. Mater. 25, 137 (2013).

[42] S. Yakunin, D. N. Dirin, L. Protesescu, M. Sytnyk, S. Tollabimazraehno, M. Humer, F. Hackl, T. Fromherz, M. I. Bodnarchuk, M. V. Kovalenko, and W. Heiss, ACS Nano 8, 12883 (2014).

[43] C. Livache, E. Izquierdo, B. Martinez, M. Dufour, D. Pierucci, S. Keuleyan, H. Cruguel, L. Becerra, J. L. Fave, H. Aubin, A. Ouerghi, E. Lacaze, M. G. Silly, B. Dubertret, S. Ithurria, and E. Lhuillier, Nano Lett. (2017).

[44] A. W. Achtstein, A. Schliwa, A. Prudnikau, M. Hardzei, M. V. Artemyev, C. Thomsen, and U. Woggon, Nano Lett. 12, 3151 (2012). 